\title{
Analysis of Carbonic Acid in Water Samples by Ion-Exclusion Chromatography with Pure Water as Eluent
}

\author{
Hui Fan, ${ }^{*}$ Mingyu Ding, ${ }^{* \dagger}$ Kazuhiko TanaKa, ${ }^{* *}$ and Wenzhi Hu${ }^{* * *}$ \\ *Department of Chemistry, Tsinghua University, Beijing, 100084, China \\ **National Institute of Advanced Industrial Science and Technology at Seto, \\ 110 Nishiibara-cho, Seto, Aichi 489-0884, Japan \\ ***Division of Chemistry, Graduate School of Science, Hokkaido University, Sapporo 060-0810, Japan
}

\begin{abstract}
A simple, sensitive and rapid ion-exclusion chromatographic method for the simultaneous separation of carbonic acid and short chain organic acids (formic acid, acetic acid, and propionic acid) has been developed. A weakly acidic cation exchange resin column TSKgel OApak-A was used for the separation; pure water, owing to its lower background conductance, was employed as eluent in order to obtain high detection sensitivity. A good separation of these weak acids was achieved in $16 \mathrm{~min}$. The linear range of the peak area calibration curve for carbonic acid was from $3.0 \mathrm{mg} / \mathrm{L}$ to 500 $\mathrm{mg} / \mathrm{L}$. The conductivity detection limit calculated at $S / N=3$ was $0.084 \mathrm{mg} / \mathrm{L}$ for carbonic acid. The method developed in this work was successfully applied to the determination of carbonic acid in several environmental water samples without any pretreatment.
\end{abstract}

(Received September 13, 2004; Accepted November 1, 2004)

\section{Introduction}

Anion exchange chromatography (AEC) with conductivity detection is the most useful simultaneous determination method for common anions such as fluoride, chloride, bromide, nitrite, nitrate, phosphate, sulfate and for some organic acids. ${ }^{1-6}$ But there are some limits in the separation or/and detection of carbonic acid $\left(\mathrm{H}_{2} \mathrm{CO}_{3}\right)$ with the AEC even though the simple and accurate determination of carbonic acid (or other inorganic carbon species, e.g., carbon dioxide, hydrogen carbonate and carbonate) is very important in water purification, environmental and biological research. ${ }^{1}$ Water is used so widely in many fields, such as power plants, heating systems, and oil fields. Carbonic acid together with other anions in water will erode the pipelines or form scale in the pipelines, which may reduce the instrument efficiency. So it is most important to analyze these anions before resolving these problems. The insufficient detection sensitivity in AEC is a limit for the determination of low-level carbonic acid in the environment water samples due to the weak acidity of carbonic acid and the higher background conductivity of eluent. Suppressed AEC with higher sensitivity cannot be used to detect carbonic acid because carbonates are usually applied as the eluent. Nonsuppressed AEC is able to separate carbonic acid, ${ }^{7}$ but the acidic eluents used commonly depress the detection sensitivity of carbonic acid by suppressing its ionization. ${ }^{8-10}$ Furthermore, on an AEC column, simultaneous separation of carbonic acid and other anions coexisting in the environmental water samples is difficult because the retention of carbonic acid is almost the same with weak acid anions such as $\mathrm{F}^{-}$, formic acid and acetic

† To whom correspondence should be addressed.

E-mail: dingmy@chem.tsinghua.edu.cn acid, and much weaker than the retention of multivalent anions such as $\mathrm{SO}_{4}^{2-}$ and $\mathrm{PO}_{4}^{3-}$.

Ion-exclusion chromatography (IEC) provides a more useful technique for the separation of organic and inorganic weak acids. Kreling ${ }^{11}$ analyzed bicarbonate in some biological fluids such as blood serum, bile, pancreatic juice and saline duodenal periusion solutions using a Dionex-HPICE-ASI column and pure water eluent. Although this method has gotten a good result, the measured bicarbonate response is not necessarily a linear function of concentration; the purity (conductivity) of the mobile phrase can also influence the observed response. Tanaka $^{12}$ determined bicarbonate by using IEC with two ion exchange enhancement columns and got a good sensitivity, but using three columns sounds a little complex.

In this research, a non-suppressed IEC method for the direct determination of carbonic acid was developed. A weakly acidic cation exchange resin column TSKgel OApak-A was used for separation and the simultaneous separation of carbonic acid; short chain organic acids (formic acid, acetic acid and propionic acid) could also be achieved on the same condition. Pure water was used as eluent, so that more high sensitivity was obtained. The determination of carbonic acid in water samples such as tap water, river water and pond water was performed. In this work, two acidic solutions used as eluents were compared with pure water, and different organic solvents added into water to improve peak shapes of organic acids are also discussed.

\section{Experimental}

\section{Apparatus}

Chromatographic analysis was carried out with a HIC-6 ion chromatograph (Shimadzu, Japan) equipped with a LP-6A pump and a CDD-6A conductivity detector, and connected to a 


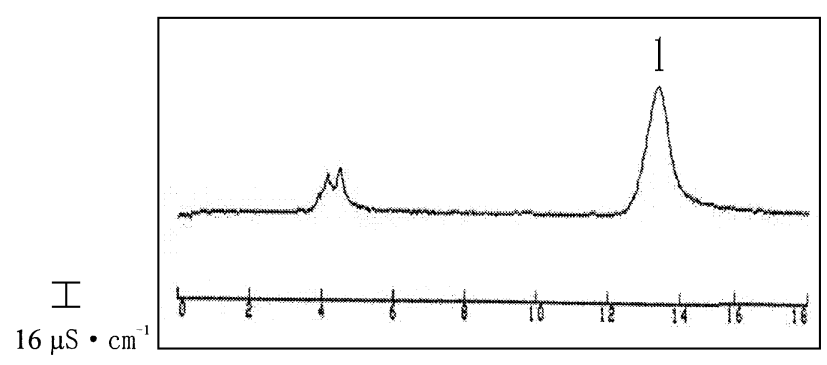

Time/min

Fig. 1 Chromatogram of carbonic acid standard obtained using pure water as eluent. Chromatographic conditions: column, TSKgel OApak-A polymethacrylate-based weakly acidic cation-exchange resin in the $\mathrm{H}^{+}$form $(300 \times 7.8 \mathrm{~mm}$ i.d. $)$; column temp., $35^{\circ} \mathrm{C}$; flowrate, $1.0 \mathrm{ml} / \mathrm{min}$; injection volume, $20 \mu \mathrm{L}$. Peak No. $1=\mathrm{CO}_{3}{ }^{2-}(60$ $\mathrm{mg} / \mathrm{L})$.

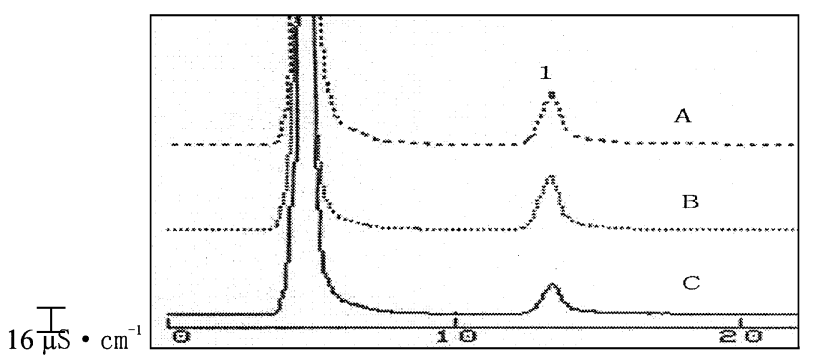

Time/min

Fig. 2 Chromatograms of the three water samples Chromatographic conditions as in Fig. 1. A, tap water; B, river water; $\mathrm{C}$, Pond water. Peak: $1, \mathrm{CO}_{3}{ }^{2-}$.

C-R4A chromatographic workstation. The separation column used is a Tosoh (Tokyo, Japan) TSKgel OApak-A $(300 \times 7.8$ $\mathrm{mm}$ i.d.) packed with a polymethacrylate-based weakly acidic cation exchange resin in the $\mathrm{H}^{+}$-form (particle size: $5 \mu \mathrm{m}$, exchange capacity: 0.1 mequiv. $/ \mathrm{mL}$ ). The selected chromatographic conditions were as follows: eluent, doubledistilled deionized water; flow rate, $1.0 \mathrm{~mL} / \mathrm{min}$; column temperature, $35^{\circ} \mathrm{C}$; and sample size, $20 \mu \mathrm{L}$ by loop.

\section{Reagents and chemicals}

All of the reagents used were of analytical-reagent grade. The carbonic acid solution was prepared by dissolving sodium carbonate in deionized water. Formic acid, acetic acid and propionic acid were prepared as aqueous solutions. All of the environmental samples including river water, pond water and tap water were taken from the campus of Tsinghua University on the same day. All of them were put at room temperature for $12 \mathrm{~h}$ so that any phytoplankton can be deposited. These samples were passed through a $0.45 \mu \mathrm{m}$ Millipore filter before injection.

\section{Results and Discussion}

Separation of carbonic acid by IEC

Under the selected chromatographic conditions, the chromatograms of carbonic acid standard and of the three water samples are shown in Figs. 1 and 2, respectively. As shown in

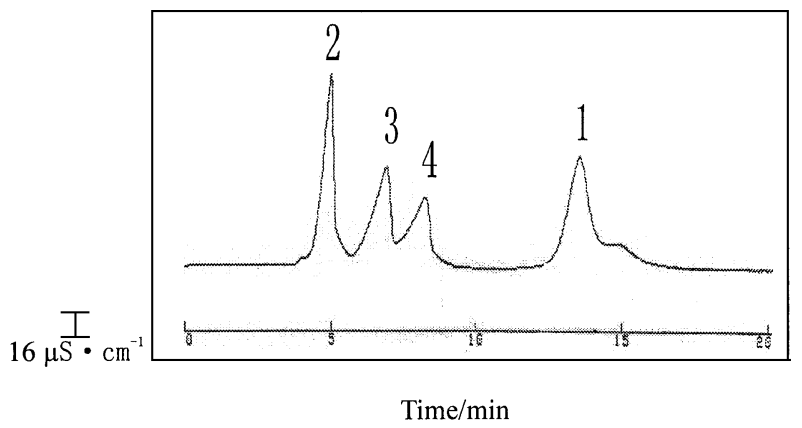

Fig. 3 Chromatogram of the four kinds of weak acid. Conditions as in Fig. 1. Peaks: $1, \mathrm{CO}_{3}{ }^{2-} ; 2$, formic acid; 3, acetic acid; 4, propionic acid.

the two figures, carbonic acid (the peak 1) has great retention on the stationary phase, with a retention time of about $14 \mathrm{~min}$, and can be separated successfully from the strong acid anions, with retention times between 4.0 and $5.5 \mathrm{~min}$. Furthermore, this method can also be used for the simultaneous separation of carbonic acid and common short chain organic acids including formic acid, acetic acid and propionic acid, as shown in Fig. 3. Although leading peaks were observed for the three organic acids, the peak shape of carbonic acid is symmetrical in all of the three chromatograms.

\section{Effects of various eluents}

For the separation of weak acids in IEC, the aqueous solution of a strong inorganic acid such as sulfuric acid or of a weak acid such as citric acid or benzoic acid, was generally used as the eluent in order to repress the ionization of the sample acids. However, the acidic eluent would cause a rather high background conductance and would reduce the detection sensitivity of weak acids when conductivity detection is used. ${ }^{9}$ Tanaka $^{14}$ used sulfuric acid solution as the eluent to separate several carboxylic acids and found that sulfuric acid eluent was very effective in the improvement of the peak shape. Use of an aqueous solution of a weak acid such as benzoic acid as the eluent could also improve the peak shapes of carboxylic acids in their studies. In this work, as shown in Fig. 3, the peaks of formic, acetic and propionic acid have tailed and broadened. In order to improve the peak shapes of these three organic acids, some acidic solutions can be used, but these electrolyte solutions would cause a higher background conductance. Three kinds of eluents: $0.1 \mathrm{~mol} / \mathrm{L}$ benzoic acid solution, $0.1 \mathrm{~mol} / \mathrm{L}$ tartaric acid solution and pure water, were compared in this work. The detection limits of carbonic acid at $S / N=3$ were 6.3 , 4.32 and $0.084 \mathrm{mg} / \mathrm{L}$ using benzoic acid solution, tartaric acid solution and pure water as eluents, respectively. Such results showed that the detection sensitivity of pure water eluent is much higher than those of the acidic eluents. So it is suitable to select pure water as the eluent for detecting carbonic acid at trace levels. Generally, if the analysis of carboxylic acids is more cared about, the acidic solutions are suitable to be used as eluents. However, the determination of carbonic acid was given more attention in this work, so pure water with the lowest background conductance was chosen as the eluent.

When pure water was used as an eluent, peaks with a leading front edge were obtained for carboxylic acids. This resulted from some adsorption as a side effect ${ }^{13}$ in IEC. To improve the peak shapes and not to enhance the background conductance so much at the same time, one can use an organic modifier. Morris and Fritz ${ }^{15}$ have found that small straight-chain alcohols added 
Table 1 Results of quantitative analysis

\begin{tabular}{|c|c|c|c|c|c|c|}
\hline \multirow{2}{*}{ Analyte } & \multirow{2}{*}{ Calibrating curve equation } & \multirow{2}{*}{$r^{2}$} & \multirow{2}{*}{$\begin{array}{c}\text { Linear range/ } \\
\mathrm{mg} \mathrm{L}^{-1}\end{array}$} & \multirow{2}{*}{$\begin{array}{l}\text { Detection limit/ } \\
\quad \mathrm{mg} \mathrm{L}^{-1}\end{array}$} & \multicolumn{2}{|c|}{$\mathrm{RSD}, \%(n=7)$} \\
\hline & & & & & Retention time & Peak area \\
\hline Formic acid & $y=1704.4 x-2233.3$ & 0.9992 & $0.5-100$ & 0.017 & 0.19 & 2.7 \\
\hline Acetic acid & $y=730.56 x+906.76$ & 0.9987 & $0.5-100$ & 0.023 & 0.28 & 2.3 \\
\hline Propionic acid & $y=483.32 x-847.36$ & 0.9994 & $5.0-800$ & 0.068 & 0.51 & 5.6 \\
\hline Carbonic acid & $y=94.825 x+300.16$ & 0.9994 & $3.0-500$ & 0.084 & 0.10 & 2.5 \\
\hline
\end{tabular}

to an eluent have a dramatic effect on the chromatographic behavior of small polar compounds such as the alkane carboxylic acids. The alcohols are believed to coat the surface of the polymeric resin owing to a dynamic equilibrium between the mobile and stationary phases. Tanaka ${ }^{14}$ also found that to enhance the methanol concentration also can reduce the retention time of weak acids. In our study, an organic modifier such as 5\% methanol, 5\% 2-propanal isopropyl alcohol or 5\% butanol was added into water. All of these organic modifiers can help a little to improve the peak shapes, but the effect is not obvious. As the concentrations of organic modifiers were increased, the background conductance was also enhanced. So the detection sensitivity of carbonic acid is also decreased. Finally, pure water was chosen as the eluent in this work.

\section{Quantitative analysis of environmental water samples}

The parameters of quantitative analysis of the four weak acids are shown in Table 1. The linear range of peak area calibration curves for all analytes was up to two orders of magnitude. The conductivity detection limits calculated at $S / N=3$ were from 0.017 to $0.084 \mathrm{mg} / \mathrm{L}$ for the four weak acids, where carbonic acid was $0.084 \mathrm{mg} / \mathrm{L}$. However, when carbonic acid was in a lower concentration (from 0.084 to $3 \mathrm{mg} / \mathrm{L}$ ), the integral areas and the concentrations were not a linear relationship. That is why the limit of quantification was much higher than the limit of detection. The same situation could be seen in the other three acids. RSDs of retention time and peak area were calculated for seven parallel experiments. Except for the peak area RSD of propionic acid which was $5.6 \%$, all of them were under $5 \%$.

The IEC method developed in this work was applied to the determination of carbonic acid in tap water, river water and pond water. All of these three kinds of water were taken from Tsinghua University on the same day. The chromatogram of the water samples is shown as Fig. 2. The big peak at about 4 min was of strong acid anions such as $\mathrm{SO}_{4}{ }^{2-}, \mathrm{NO}_{3}{ }^{-}$, and $\mathrm{Cl}^{-}$, which were not separated from each other. Carbonic acid in water can be detected without influence of these anions. If some organic acids which appeared at about 5-12 min coexisted in water, they would not influence the analysis of carbonic acid. The carbonic acid contents were $341.1 \mathrm{mg} / \mathrm{L}$ in tap water, $169.2 \mathrm{mg} / \mathrm{L}$ in river water and $336.2 \mathrm{mg} / \mathrm{L}$ in pond water, respectively.

\section{Conclusions}

In this study, an IEC method for the determination of carbonic acid was investigated using a weakly acidic cation-exchange resin. A good resolution and highly sensitive detection of carbonic acid were accomplished by eluting with pure water. It was possible to separate several organic acids and carbonic acid at the same time. In this study, pure water was applied as the eluent, so the peak shapes of carboxylic acids were not so symmetric.

The proposed method is easier and quicker than the previous works and it has a much lower detection limit, a much wider linear range and a good repeatability. This method has been successfully applied to the determination of carbonic acid in some water samples such as tap water, river water and pond water. And it can also be adopted in many other natural water sample analysis.

\section{References}

1. M. Zenki, T. Nabekura, and A. Kobayashi, Analyst, 1993, 118, 273.

2. A. Alcázar, Fernández-Cáceres, M. J. Martín, F. Pablos, and A. G. González, Talanta, 2003, 61, 95.

3. Z. Lu, Y. Liu, V. Barreto, C. Pohl, N. Avdalovic, R. Joyce, and B. Newton, J. Chromatogr., A, 2002, 956, 129.

4. G. Schminke and A. Seubert, J. Chromatogr., A, 2000, 890 , 295.

5. J. A. Morales, L. S. de Graterol, and J. Mesa, J. Chromatogr., A, 2000, 884, 185.

6. A. Caliamanis, M. J. McCormick, and P. D. Carpenter, Anal. Chem., 1999, 71, 741.

7. S. Charbonneau, R. Gilbert, and L. Lépine, Anal. Chem., 1995, 67, 1204

8. V. T. Turkelson and M. Richards, Anal. Chem., 1978, 50, 1420.

9. P. R. Haddad and P. E. Jackson, "Ion ChromatographyPrinciples and Applications", 1990, Elsevier, Amsterdam.

10. K. Tananka, K. Ohta, and J. S. Fritz, J. Chromatogr., A, 1997, 770, 211.

11. J. R. Kreling and J. DeZwaan, Anal. Chem., 1986, 58, 3028.

12. K. Tanaka and J. S. Fritz, Anal. Chem., 1987, 59, 708.

13. K. Tanaka, K. Ohta, J. S. Fritz, Y-S. Lee, and S-B. Shim, J. Chromatogr., A, 1995, 706, 385.

14. K. Tanaka, H. Chikara, W. Hu, and K. Hasebe, J. Chromatogr., A, 1999, 850, 187.

15. J. Morris and J. S. Fritz, Anal. Chem., 1994, 66, 2390. 